\title{
Hydrogen Optical Fiber Sensors
}

\section{Final Report}

\author{
July 28, 2008
}

\author{
Contract Information \\ Contract No.: DE-FG36-06G086057 \\ Period of Performance: 05/01/06 to 04/30/08
}

Presented to:

U.S. Department of Energy

Golden, CO

Technical Monitor:

James Alkire

(303) 275-4795

\author{
Presented by: \\ Intelligent Optical Systems, Inc. \\ 2520 West 237th Street \\ Torrance, California 90505 \\ Principal Investigator: \\ Robert A. Lieberman, Ph.D.
}

(424) 263-6310

\begin{abstract}
The information in this report and any hardware delivered in conformity with Grant DEFG36-06G086057 is the property of Intelligent Optical Systems, Inc., as set forth in 10 CFR 600.325 Appendix A, Patent Rights (Small Business Firms and Nonprofit Organizations (OCT 2003).
\end{abstract}




\section{EXECUTIVE SUMMARY}

During the past year this project focused on the development of, and solution to, several technical hurdles involving optrode-based hydrogen sensors. An optrode (a contraction of "optical" and "electrode") consists of a porous substrate - typically glass - that is optically transparent and into which sensor chemistry can be deposited. The optrode is placed in a location where leak detection is important and it is connected to a detector via optical fiber. A pulse of light is sent down the optical fiber to the optrode, and the intensity of the return light signal indicates whether hydrogen is present, and how much. This technology has advantages over conventional methods for detecting flammable gases such as hydrogen, because the optrode doesn't require an electric current to operate. That means it cannot act as a source of ignition. Optrodes are also less costly to fabricate, which may be important as hydrogen and fuel cell technologies move closer to mass production.

Two of the challenges presented by optrode based hydrogen sensors are signal loss due to the presence of oxygen, and also due to moisture or humidity. Oxygen appears to react with hydrogen in the presence of hydrogen sensor chemistries to produce water vapor. This effect is minimal at higher hydrogen concentrations, but can be very important at the concentrations that must be measured for effective leak detection. Moisture acts differently. It is attracted to the glass of the optrode, changing its optical properties, and also slows down the sensor response.

Major progress was made in addressing both these issues by developing new sensor chemistries, introducing hydrophobic materials to reduce the humidity effect, and exploring new substrate materials as alternatives to the porous glass. An optrode formulation was developed using a novel two-layer polymer composite to inhibit humidity interference. This was combined with a sensing chemistry that functions well in the presence of oxygen. Resulting tests showed that the sensor continued to respond well to $\mathrm{H}_{2}$ in air at 95\% relative humidity (i.e., our coating significantly reduced the interference effects of both humidity and oxygen). This dramatic improvement indicates that additional work on this polymer system can further improve the signal-to-noise ratio of this prototype sensor, yielding an easily distinguishable response to $1 \%$ hydrogen in an environment containing oxygen and over normal ranges of humidity.

The polymer film work led to a new avenue of exploration, namely eliminating the porous glass optrode and embedding the hydrogen sensor chemistry directly in the polymer as a thin film sensor. This opens a wider range of potential applications, from extended optical fiber sensing networks for buildings and garages to simple plastic "stickers" that change color when hydrogen is present at an unsafe concentration.

As hydrogen moves out of industry and commerce and into the mainstream (fuel cell cars from Honda, BMW, and General Motors; hydrogen-powered city buses being deployed around the world; replacements for nickel metal hydride batteries in video cameras, etc.) new types of sensors will be required to address the needs of these new applications. These sensors will need to conform to their individual applications and provide an unambiguous signal understandable to the lay public with no particular or specialized training. This program is addressing these emerging needs. 


\subsection{INTRODUCTION}

This final report summarizes the work conducted by Intelligent Optical Systems, Inc. (IOS) between May 1, 2006 and April 30, 2008 under the contract entitled "Hydrogen Optical Fiber Sensors" (Contract No. DE-FG36-06GO86057).

The hazards associated with using a combustible gas for fuel purposes can be reduced with sensor technology that can monitor the gas supply and warn in the event of a release. The scope of this project is to further develop IOS's proprietary hydrogen sensitive chemical formulation into optical based hydrogen sensors that can be used either with intrinsically safe remote fiber optic networks, or in compact (handheld or wall mountable) sensor units.

\subsection{TECHNICAL RESULTS}

\section{$2.1 \quad$ Background}

IOS's chemical formulation was originally infused into a porous glass substrate. Commercialoff-the-shelf porous glasses often exhibit properties, such as high oxygen permeability and hydrophilicity that are less than optimal for metal-catalyzed hydrogen sensing. The primary objective of this research is to reduce or eliminate the interference, caused by humidity and oxygen, exhibited by virtually all current optically-based hydrogen detectors.

\section{$2.2 \quad$ Tasks}

The tasks to be performed were detailed in the statement of project objectives and are summarized as follows:

1. Acquire reagents and substrate materials

2. Formulate sol-gel sensors from silicate and/or silicone reagents

3. Evaluate hydrogen diffusion in polymer materials

4. Evaluate various techniques to produce thin-film and/or slab sensors from advanced polymers and fabricate candidate sensors

5. Evaluate sensor response to hydrogen under inert conditions

6. Evaluate sensor performance and resistance to moisture and oxygen

7. Incorporate new sensors in compact hydrogen detector and test system

8. Project management and reporting

New indicator-immobilization methods, including sol-gel based immobilization, were studied. Polymers were evaluated as a substrate for immobilization, and for providing a barrier to oxygen and moisture. An assessment of the optimized sensor materials was performed. A compact hydrogen detection system, which includes advanced data acquisition and processing, was developed.

\section{$2.3 \quad$ Results}

\subsubsection{Equipment and Supply Acquisition}

We acquired commercial porous glass to evaluate our sensor chemistry with advanced polymeric barrier moisture materials to achieve optimum performance at elevated humidity levels. Concurrently, we acquired several different types of metal nanoparticles to be tested as a replacement for our currently used metal catalyst. Optical grade polymers were acquired to test 
and optimize the transformation of our hydrogen sensor chemistry into a high performance integrated waveguide format.

\subsubsection{Device Testing Apparatus}

A hydrogen testing apparatus, shown schematically in Figure 1, was assembled. The testing system, which is used for sensor material optimization, is described as follows: The samples are held in a gas flow cell in a manner that allows them to be optically monitored. Hydrogen test concentrations are obtained by mixing certified gas with air using computer-controlled digital mass flow controllers (MFC). The test gas can be humidified between 0-95\% RH. A solenoid valve is used to direct the gas into the gas flow cell. Broadband (white) light is delivered to the flow cell, and is collected by optical fibers and carried back to the detection unit. Changes in the presence of hydrogen cause changes in optical absorbance, which the detection unit transmits to the computer. The detection unit is capable of fast continuous data acquisition, which allows us to measure and record the hydrogen sensor response in real time.

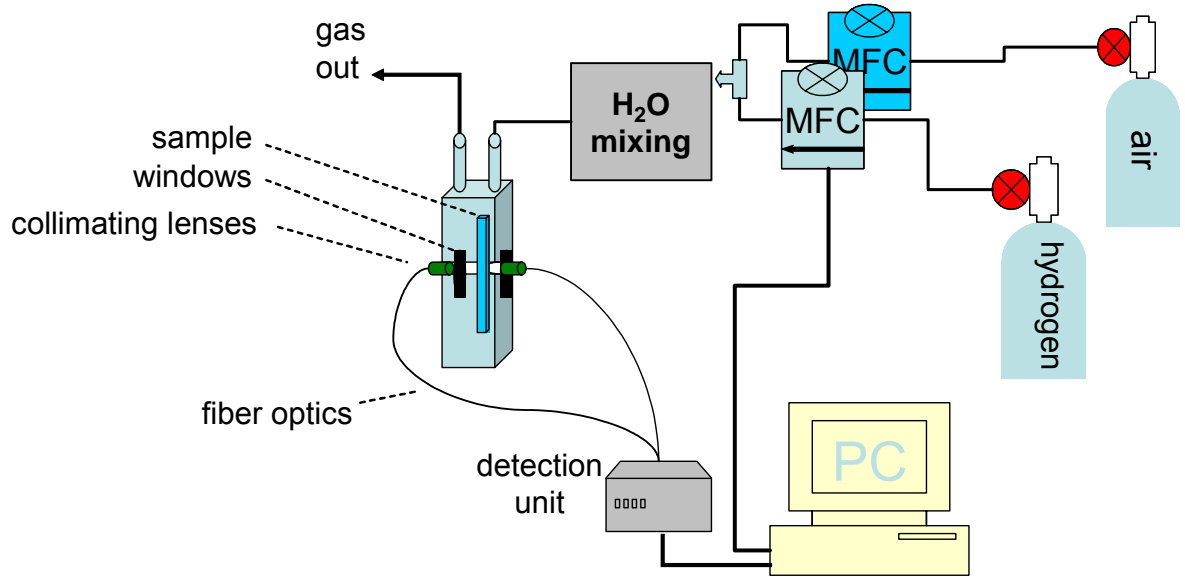

Figure 1 Schematic of IOS sensor testing system.

\section{Multiplexed Detection Apparatus}

We developed a prototype multi-channel fiber optic detection unit, as shown in Figure 2. This unit has a small footprint and can be readily combined with the final eight-channel sensor design. The unit consists of a proprietary optoelectronic and software system that uses LEDs and solid state detectors. A 9 volt DC source powers the proprietary electronics, which are designed to be used in a portable multiplexed format, allowing up to eight sensors to be monitored simultaneously, in real time, using fiber optic cables. 


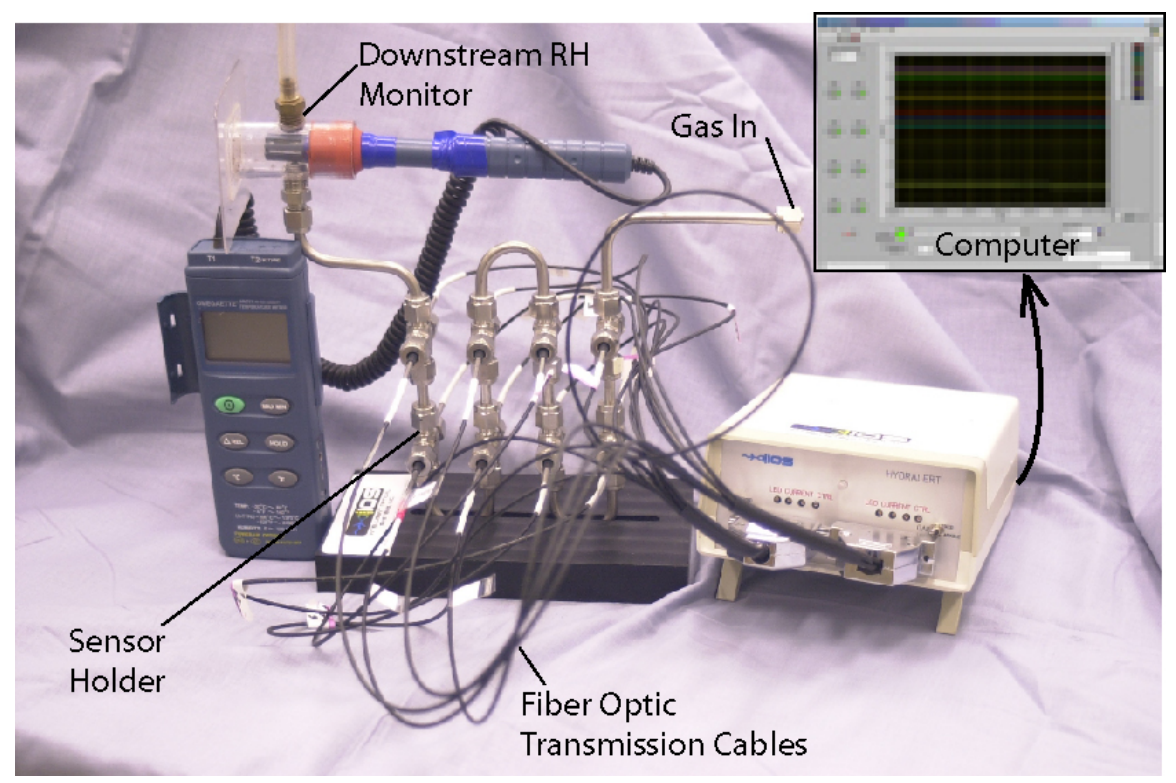

Figure 2 Multi-channel fiber optic detection unit controlled by personal computer using operator friendly graphical interface (inset).

The unit is controlled by a personal computer (PC) using software written in Labview ${ }^{\circledR}$. The software includes an easy-to-use graphical user interface (GUI), which is illustrated in the Figure 3 inset. Data is streamed to the computer via a RS-232 serial interface.

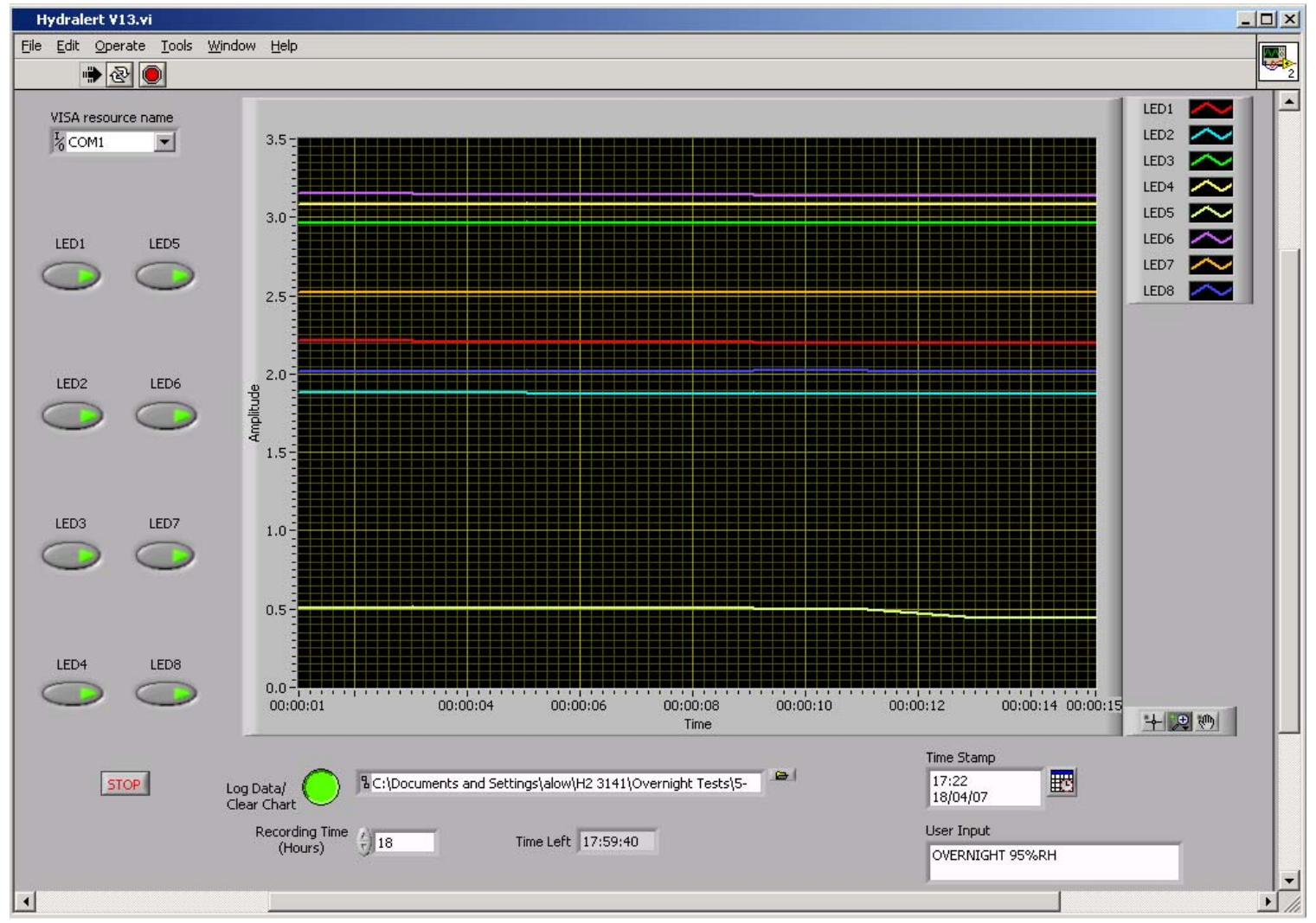

Figure $3 \quad$ Graphical user interface for 8-channel sensor testing apparatus. 
A sample set of data (exported as a bitmap image), which shows the monitored signals from eight porous glass sensors being exposed simultaneously to on and off cycles of $4 \%$ hydrogen, is shown in Figure 4. This unit is a first-generation device that can be used to simultaneously monitor up to eight sensors in different locations. This unit can be upgraded into a more sophisticated detection device that could be miniaturized further to a smaller footprint with battery power and lock-in detection. Such a device would be suitable for use in a real-world multipoint hydrogen detection system.

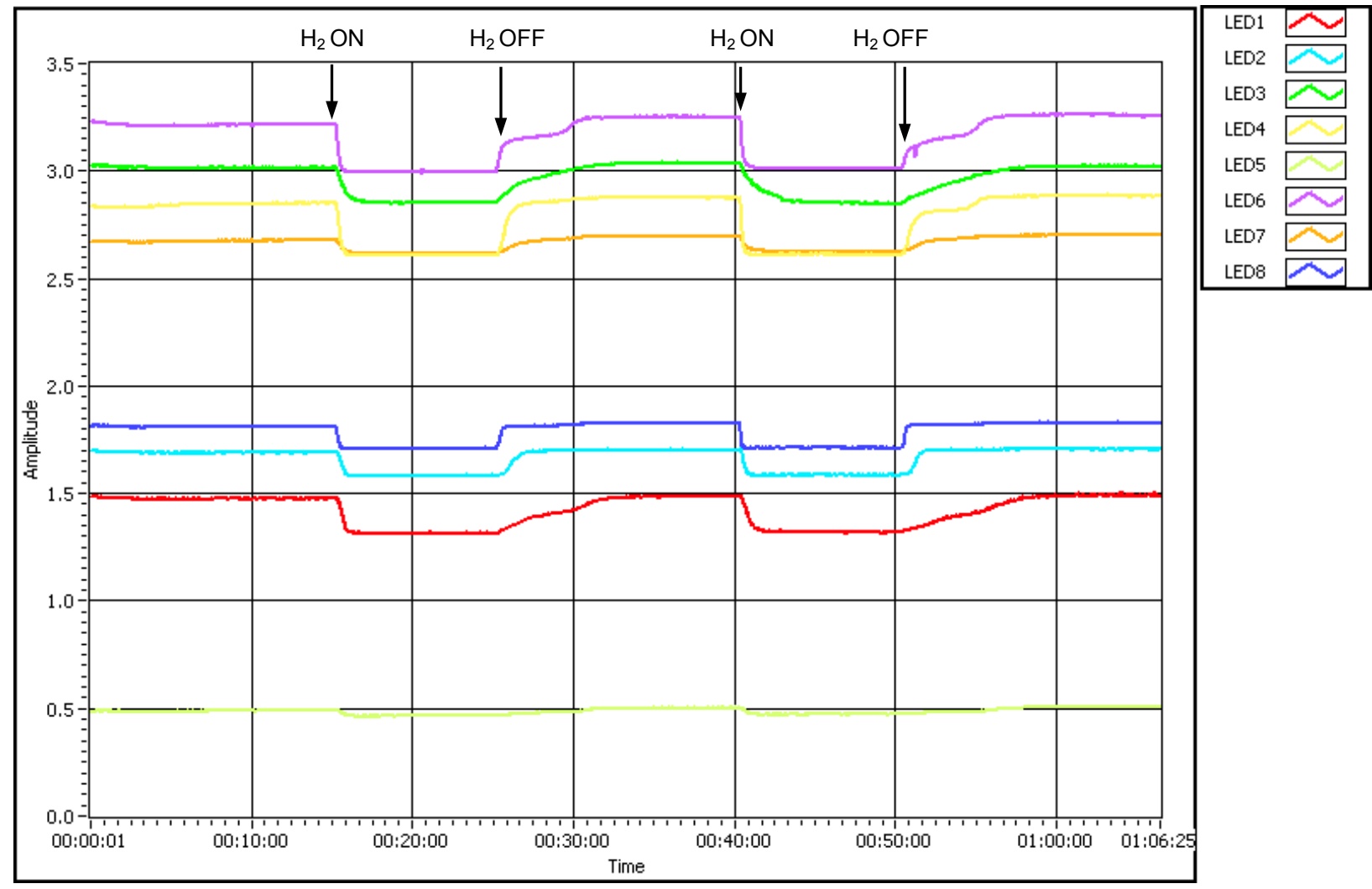

Figure 4 Data exported from the test unit as a bitmap image shows the time resolved monitoring of eight different hydrogen sensors. Hydrogen $(4 \%)$ is introduced and removed at the labeled times.

\subsubsection{Sensor Fabrication and Optimization}

Four different proprietary sensor chemistries, designated as "A-D" in the following discussion, have been used to formulate novel combinations of catalysts, indicators, and matrices. These formulations were evaluated for their ability to detect hydrogen without interference from water and oxygen. Two sensor formats have been developed: one is a porous glass optrode with a polymeric vapor barrier coating; the second is a thin film made using advanced polymers.

\section{Sol-Gel Matrix}

We synthesized a variety of sol-gel based sensor materials using catalyst/indicator chemistry "B". Due to the low permeability of hydrogen into our silicon-based sol-gel structures, the response and recovery times were far too long for these materials to be useful as either thick film or monolithic sensors. Thin films show much better response times, but the thin film preparation route has been highly problematic. Controlling the film thickness is difficult, and curing effects often leave the underlying substrate warped. As a solution to the limited porosity of the sol-gel 
glasses we synthesized, we investigated a method in which we pulverized the sol-gel glasses into micrometer-sized particles. These particles were then blended with a silicone polymer and cast into a thin film. This methodology works to sense hydrogen, but does not yield fast response times (see Figure 5). We determined that fast response times can only be achieved with films of sol-gel (or sol-gel/polymer), on the order of 5 microns thick or less. However, the signal produced via optical transmission measurements is constrained using such a short pathlength. Since it was not cost effective to increase the pathlength by integrating these films into a waveguide format, we concluded this portion of the project.

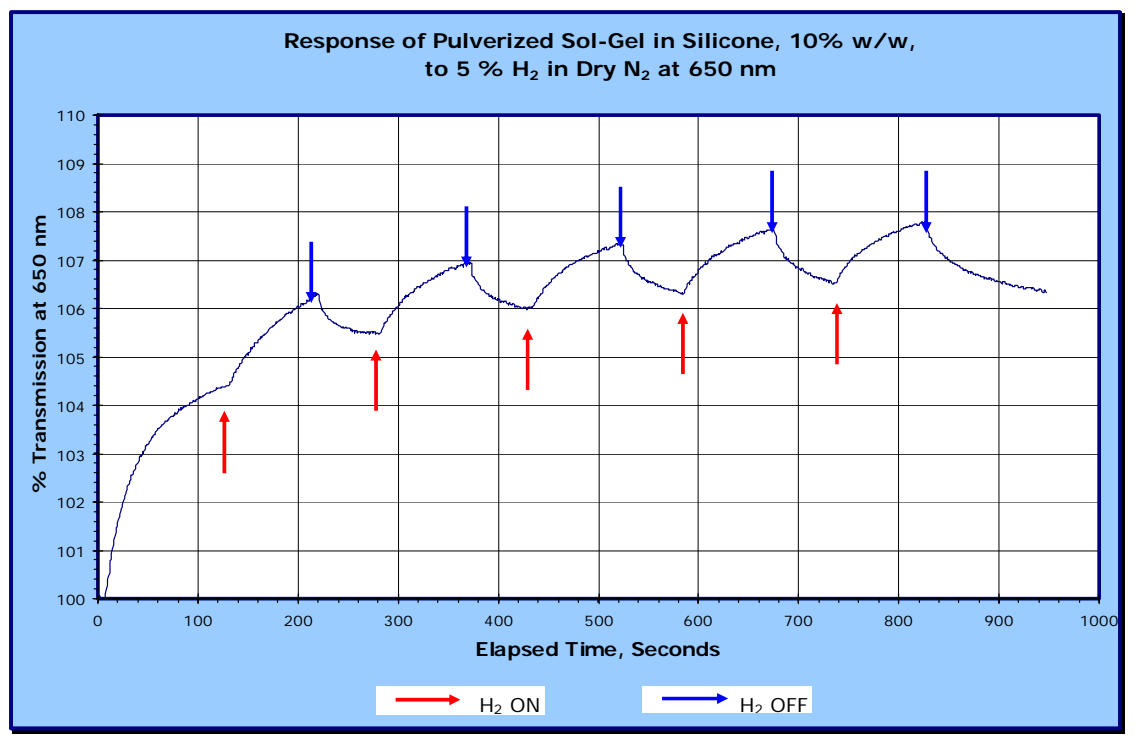

Figure 5 Transmission intensity of 650nm light taken from a thin film (25 microns) made up of pulverized sol-gel material uniformly dispersed in a hydrophobic silicone polymer. The sensor material is exposed to $5 \% \mathrm{H}_{2}$ cycling and shows a rather slow response and recovery.

Alternatively, it was determined that non-traditional sol-gels could be used instead of siliconbased sol-gel substrates. To that end, we successfully immobilized catalyst/indicator chemistry "A" in an inorganic nanostructured film that was 3 microns thick. The porous film shows a fast colorimetric response upon exposure to $5 \% \mathrm{H}_{2}$ in dry air, and has a fast recovery rate (see Figure 6). The response was reproducible during the on-off cycling of $\mathrm{H}_{2}$. 


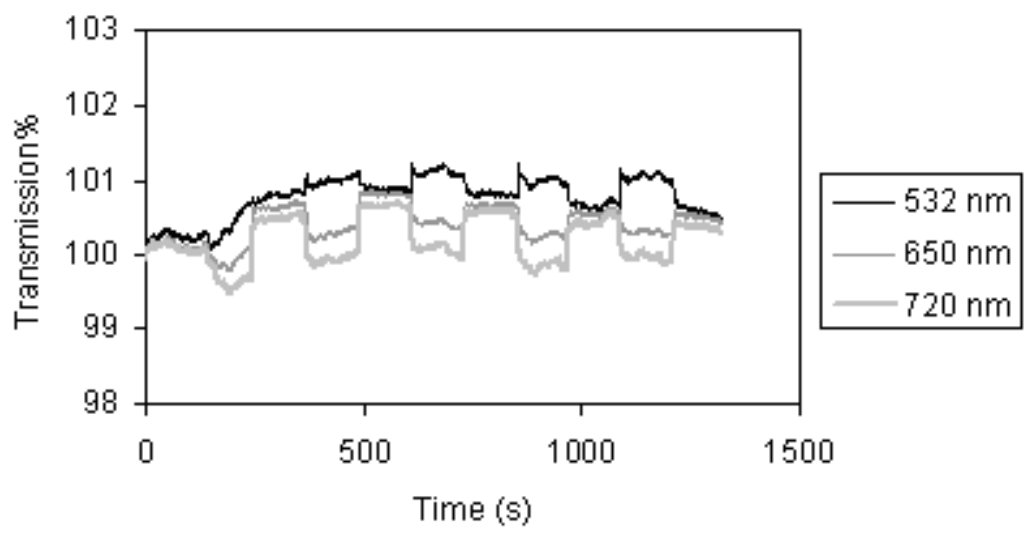

Figure 6 Thin film exposed to $5 \% \mathrm{H}_{2}$ cycling shows colorimetric response. The $532 \mathrm{~nm}$ wavelength shows an increase of light transmission while the longer $650 \mathrm{~nm}$ and $720 \mathrm{~nm}$ wavelengths showed a decrease in light transmission.

\section{Porous Glass Matrix with Vapor Barrier}

We prepared porous glass sensors using chemistries "B" and "C," so that we could use them to test polymeric vapor barrier materials. We evaluated several polymeric materials know for their hydrophobic nature. These materials were coated over the porous glass substrates. The substrates were then exposed to hydrogen in various humid backgrounds in order to test the interference of water vapor and oxygen on the response of the sensors.

We started with sensors containing chemistry "B" and having no polymer coating. These were first exposed to $5 \% \mathrm{H}_{2}$ in either a dry $\mathrm{N}_{2}$ or dry air background. Next, they were exposed to $5 \%$ $\mathrm{H}_{2}$ in either humidified $\mathrm{N}_{2}$ or humidified air. The results, presented in Figure 7, reveal that the change in sensor signal value is greater in the presence of humidity for chemistry "B." Furthermore, the drift from baseline is practically non-existent in the presence of both oxygen and humidity. This indicates that both water and oxygen are critical to the sensitivity of this chemistry, which correlates with real-life situations. 
Dry $\mathrm{N}_{2}$

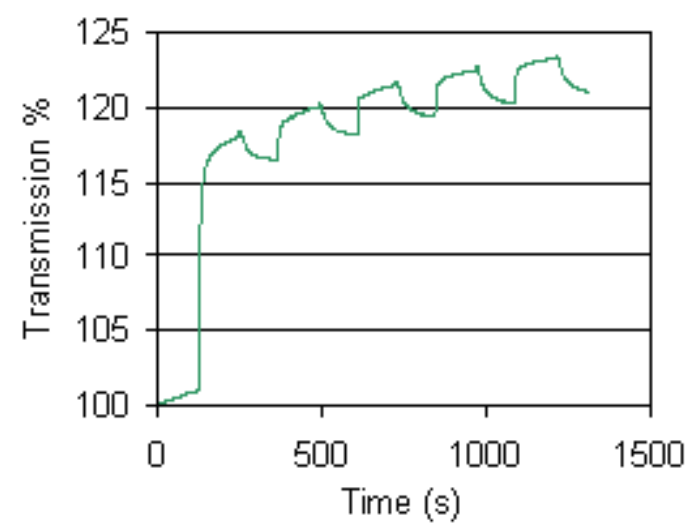

Humid $\mathrm{N}_{2}(10 \% \mathrm{RH})$

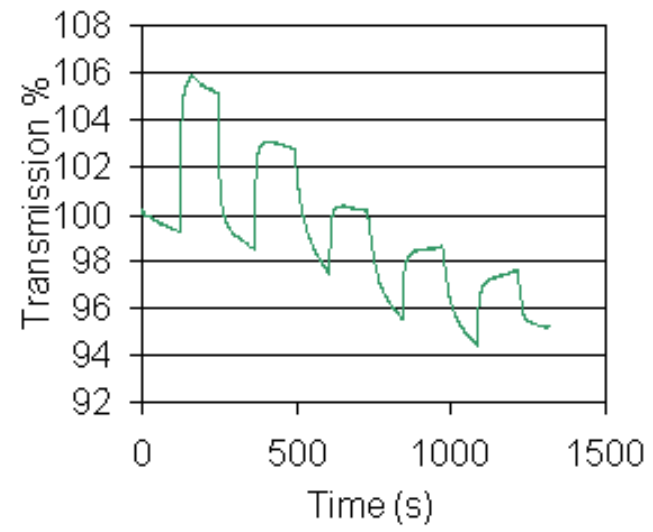

Dry air

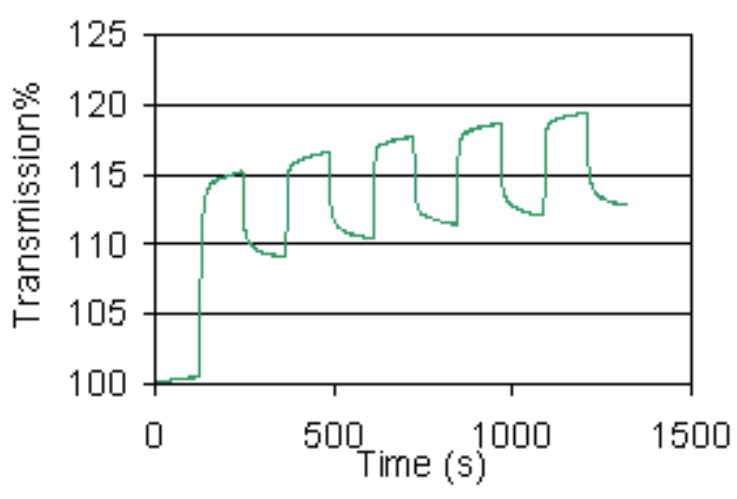

Humid air (10\%RH)

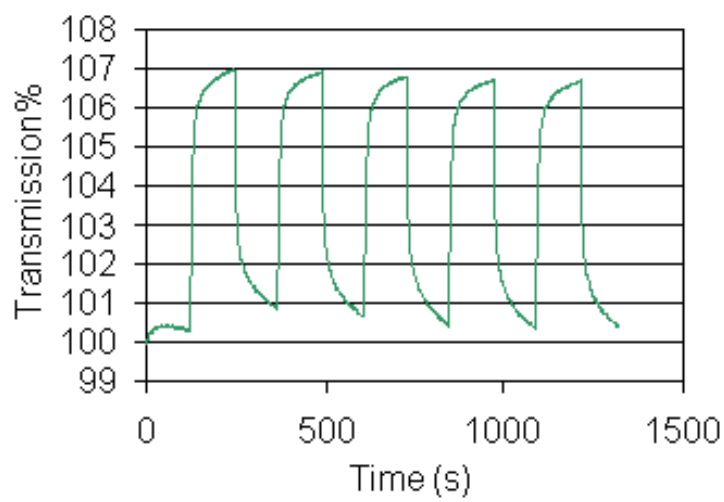

Figure $7 \quad$ The sensor response to cyclic exposures of $\mathrm{H}_{2}$ in various environments.

In attempts to improve the stability of these sensors, they were coated with a thin layer of a proprietary polymer barrier material, designated as "P-1". Since moisture plays an important role in sensitivity, the coating is used to control the humidity without causing the baseline to shift, or the sensor to lose sensitivity. At the low levels of humidity used for the initial tests, it was apparent that the barrier worked to stabilize baseline drift in the $\mathrm{N}_{2}$ background experiments (see Figure 8). However, this level of humidity does not affect the sensor in the presence of oxygen. 

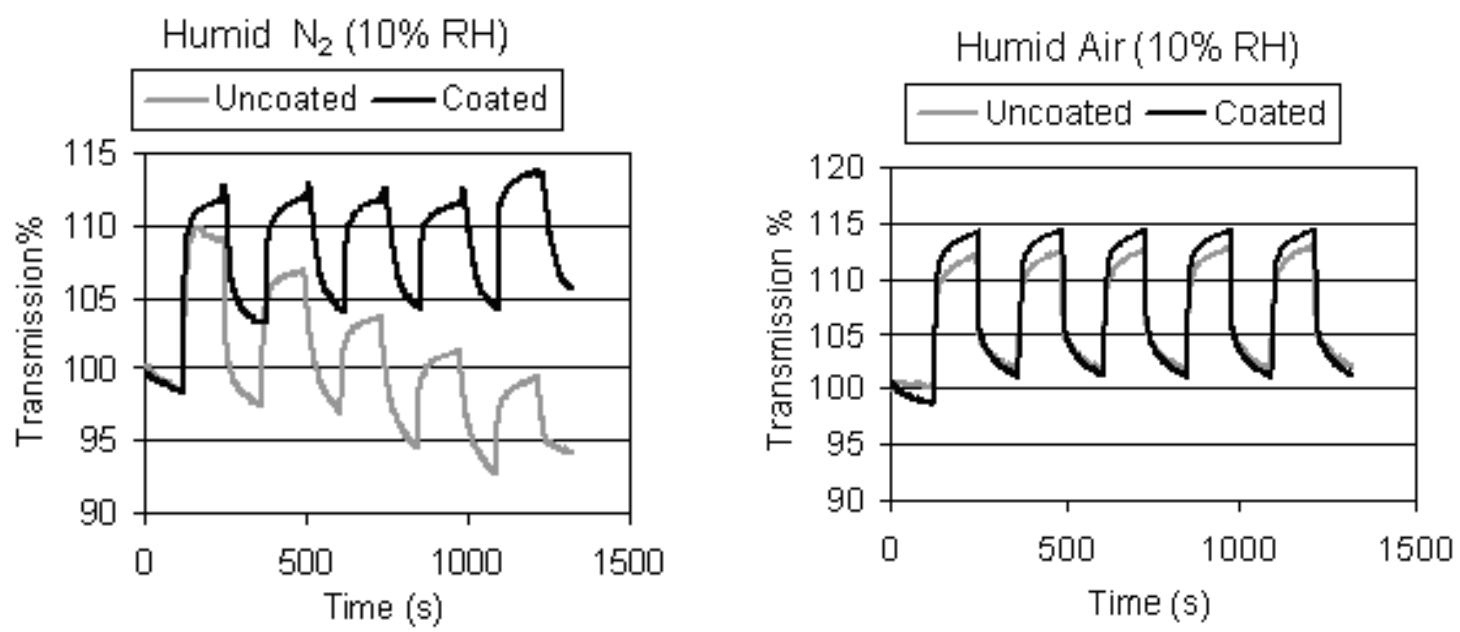

Figure 8 Coated and uncoated sensor response to cyclic exposures of $\mathrm{H}_{2}$ in various environments

Porous glass sensors prepared using chemistry "C" and polymer barrier "P-1" were evaluated. These sensors were cyclically exposed to varying concentrations of $\mathrm{H}_{2}$; the results are shown in Figure 9. The "P-1" barrier slows the diffusion of hydrogen (as evidenced by the slower response and recovery times). The sensitivity, however, appears to be unaffected.
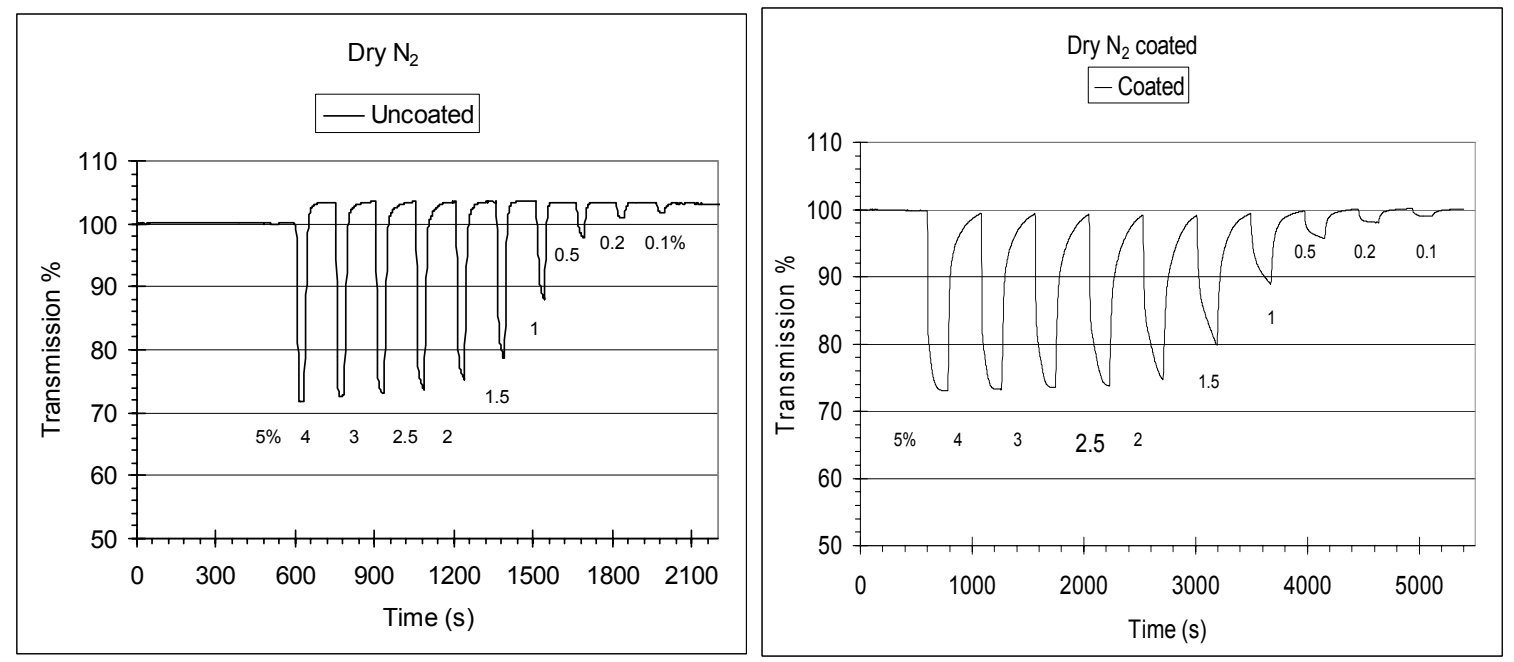

Figure 9 The coated and uncoated sensor response to cyclic exposures of $\mathrm{H}_{2}$ in an inert atmosphere. The plots are labeled with the concentrations of $\mathrm{H}_{2}$ that were tested.

We continued to evaluate polymeric materials known for their hydrophobic nature. These materials are absorbed into the porous glass substrates. The substrates are then exposed to hydrogen in various humid backgrounds to test the interference of water vapor. The results, shown in Figures 10(a) and 10(b), show the advantage of the moisture barrier. The moisture barrier stabilizes the sensor drift that is incurred in the presence of humidity, but requires further optimization. 


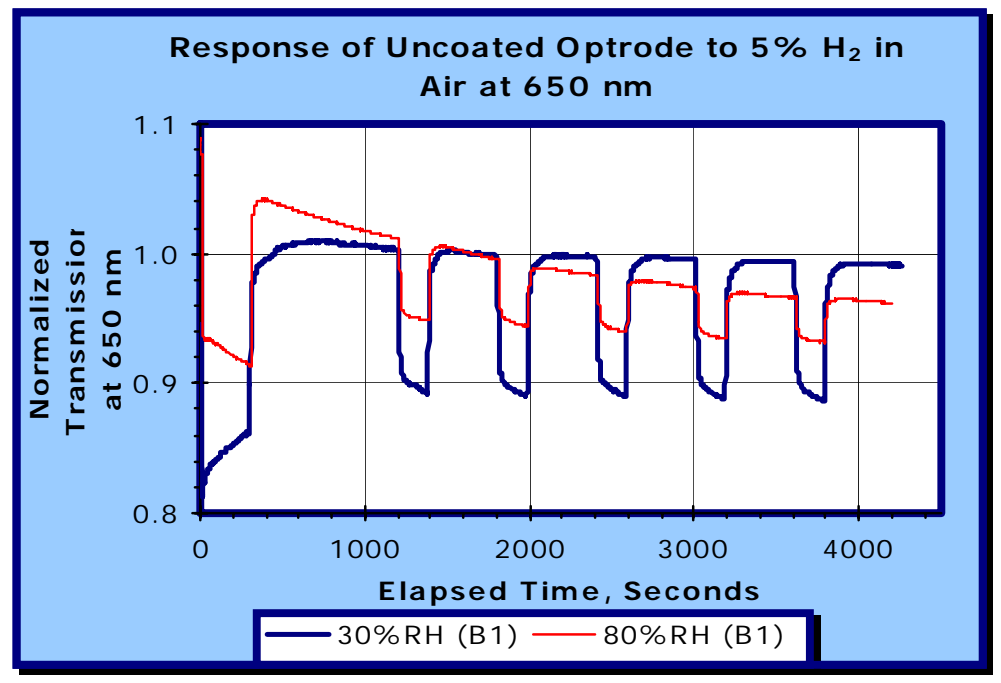

(a)

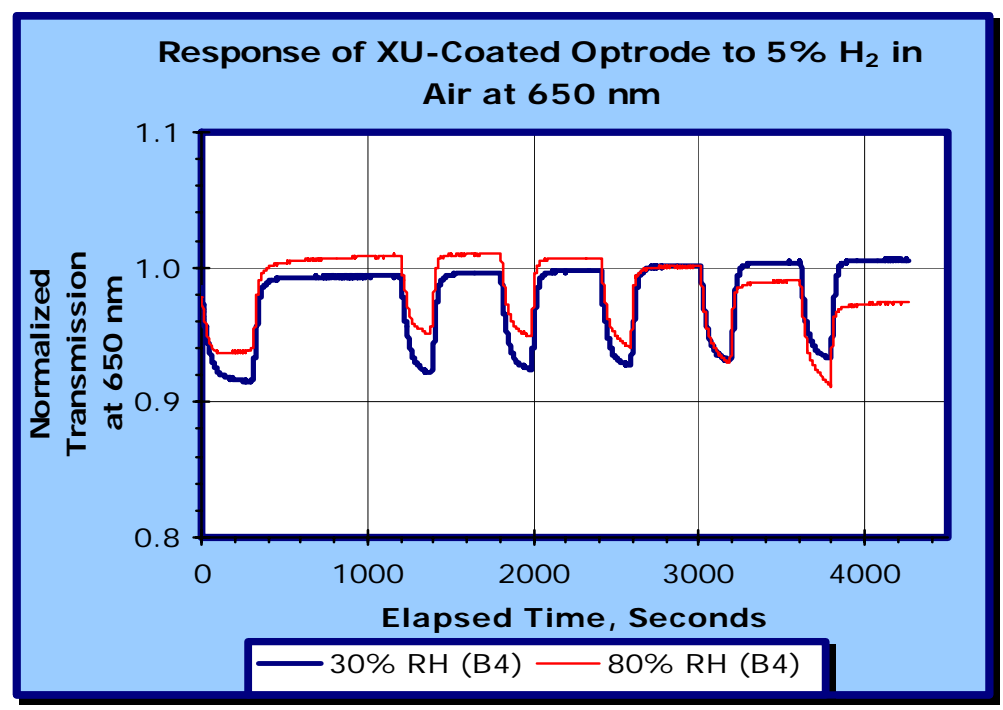

(b)

Figure 10 Uncoated (a) and coated (b) sensor response to cyclic exposures of $5 \% \mathrm{H}_{2}$ in $30 \%$ and $80 \% \mathrm{RH}$ environments.

The coated sensor substrates were pre-conditioned to a determined level of humidity for twelve hours, after which they were exposed to two on and off cycles of $4 \%$ hydrogen so that we could determine preliminary humidity interference. Four percent hydrogen was chosen because it is the lower flammable limit (LFL) of hydrogen, thus it is necessary to detect this level for indoor safety applications. The humidity levels used were $0,25,50,75,95$, and $100 \% \mathrm{RH}$. The effect of humidity on a coated sensor versus an uncoated sensor is shown in Figure 11. The response to hydrogen is influenced by the humidity level much more so for the uncoated sensor, showing the advantage of using the moisture barriers. In fact, the uncoated sensor loses its response to hydrogen when it undergoes prolonged exposure to humidity levels that exceed $75 \% \mathrm{RH}$, but the coated sensor continues to respond up to $100 \% \mathrm{RH}$. In performing these initial experiments, we observed that the sensitivity decreases by $60 \%$ as the humidity increases to $100 \%$. Figure 12 
shows a plot of the sensitivity versus the humidity level for both coated and uncoated porous glass sensors. The sensitivity is represented as the normalized change in the percent transmission before and after hydrogen exposure, as extracted from Figure 11. The moisture barrier greatly reduces the interference incurred in the presence of humidity. The polymer coatings will undoubtedly increase operational life by minimizing the poisoning effects that atmospheric contents have on the metal catalyst.
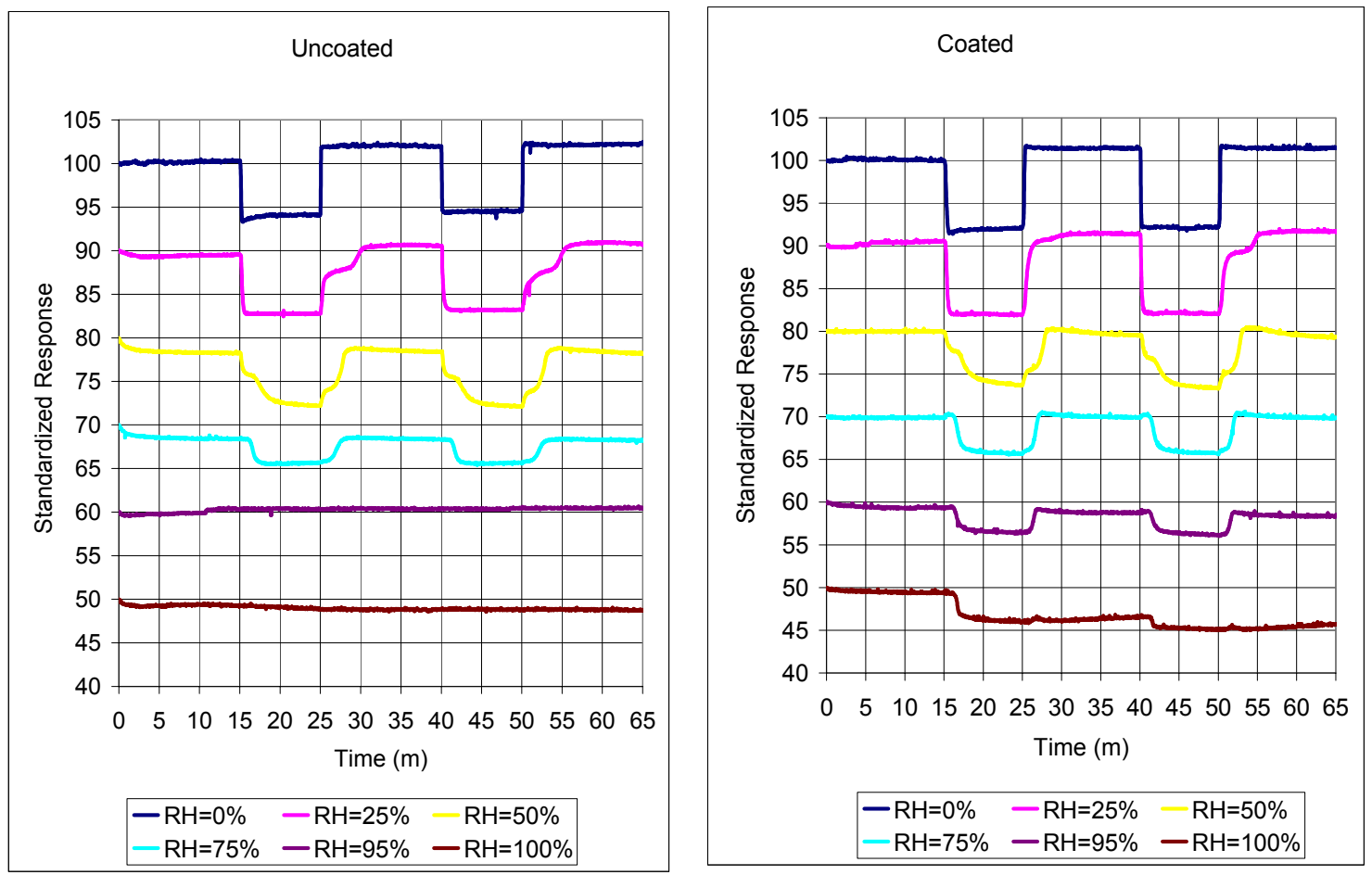

Figure 11 Uncoated (left) and coated (right) sensors response to two on and off cycles of $4 \%$ $\mathrm{H}_{2}$ in $0,25,50,75,95$, and $100 \% \mathrm{RH}$ environments. 


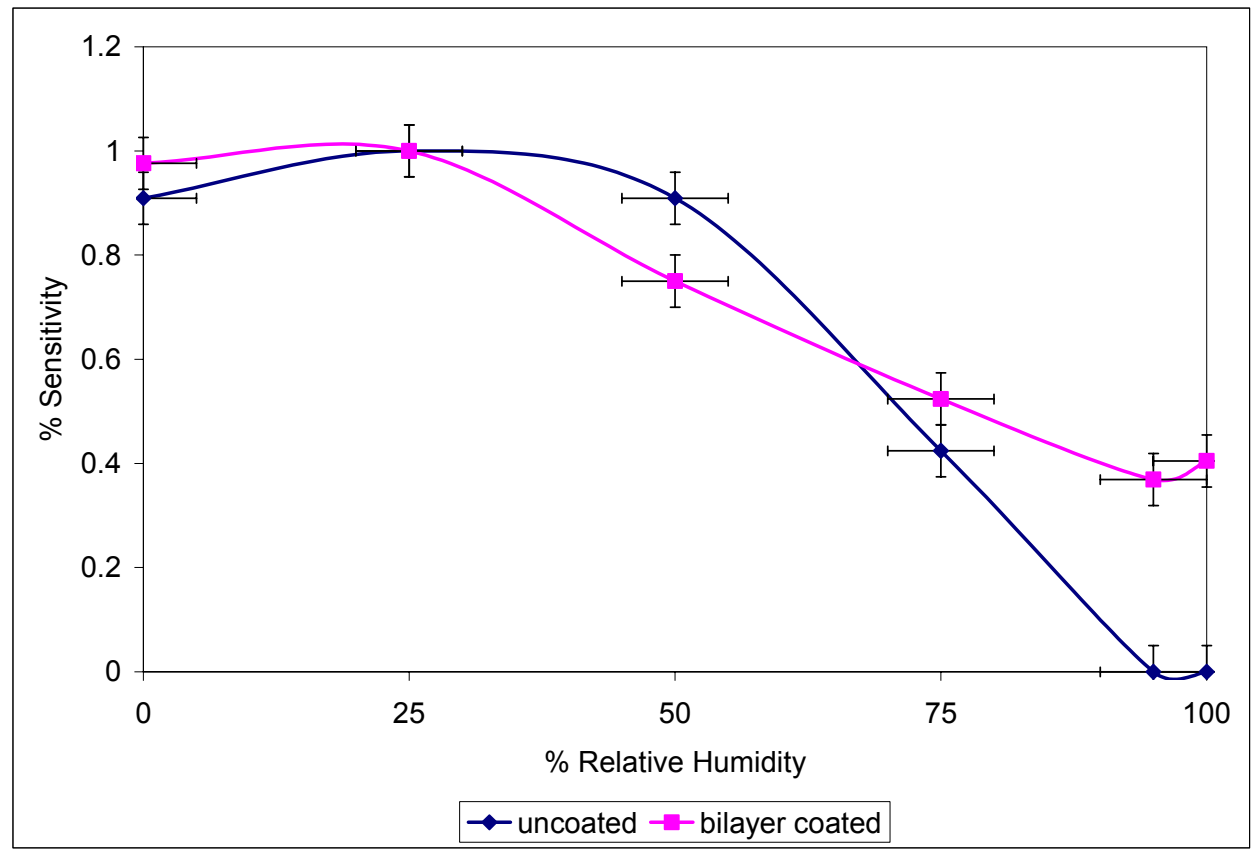

Figure 12 Effect of prolonged exposure to humidity on a coated versus uncoated sensor. The uncoated sensor loses its properties when the humidity is greater than $75 \%$. The coated sensor remains useful up to $100 \% \mathrm{RH}$.

The protocol for coating porous glass substrates with polymeric moisture barriers was optimized to include a two-layer coating process. We observed that no single polymer performs adequately as a barrier at elevated humidity, but we can combine two layers of differing polymers to get the characteristics we desire. The polymers are first dissolved in solvent, and the solutions are then imbibed in the porous glass sensor, one at a time, with a solvent evaporation step between.

The performance of a representative porous glass sensor coated with a novel polymer composite is shown in Figure 13. This data shows that the sensor continues to respond to $\mathrm{H}_{2}$ at $95 \% \mathrm{RH}$ when oxygen is present in the environment (i.e., our coating significantly reduces the interference effects of both humidity and oxygen). This dramatic improvement indicates that additional work on this polymer system can further improve the signal-to-noise ratio of this prototype sensor, yielding an easily distinguishable response to $1 \%$ hydrogen in an environment containing oxygen. The artifacts in the response data graphs, which cause the appearance of drift and signal fluctuation, were induced by our gas mixing setup. The source of these artifacts has been identified and will be eliminated in future work. 


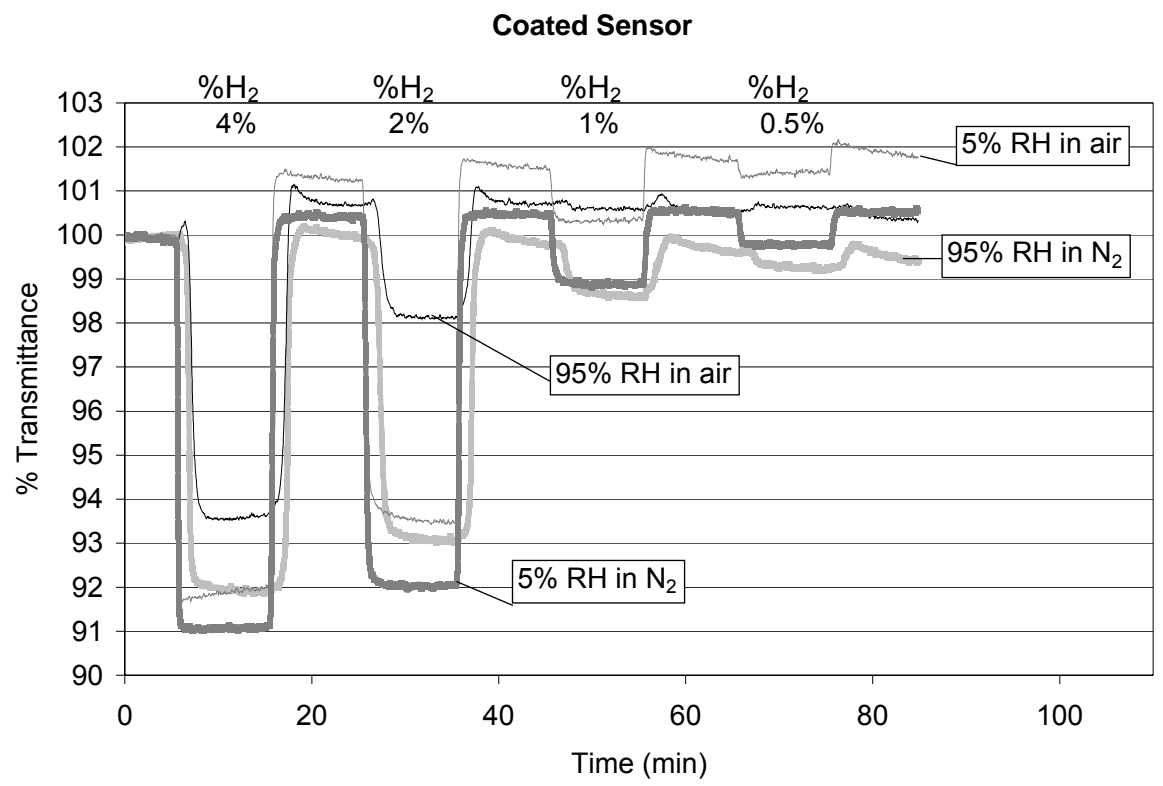

Figure 13 Effect of relative humidity and environment on coated sensor response to hydrogen cycling of 4,2,1,0.5\% (measured as sample transmittance of $650 \mathrm{~nm}$ light).

\section{Polymer Matrix}

Solution-based hydrogen sensor chemistries are known to be difficult to immobilize in polymeric substrates. IOS, however, has shown the feasibility of using this approach to fabricate thin polymeric sensor films using chemistry "D". The initial polymer that we used is a proprietary formula ("P-2") that has excellent optical clarity and film forming properties. The thin film was exposed to 5\%, 4\%, 3\%, 2\%, and 1\% hydrogen in both humid $\mathrm{N}_{2}$ and humid air (Figure 14). This initial sensor shows a slight drift in the baseline (more so under humid $\mathrm{N}_{2}$ conditions). It is important to note that the $20 \mu \mathrm{m}$ thin sensor film responds easily to $1 \%$ hydrogen.

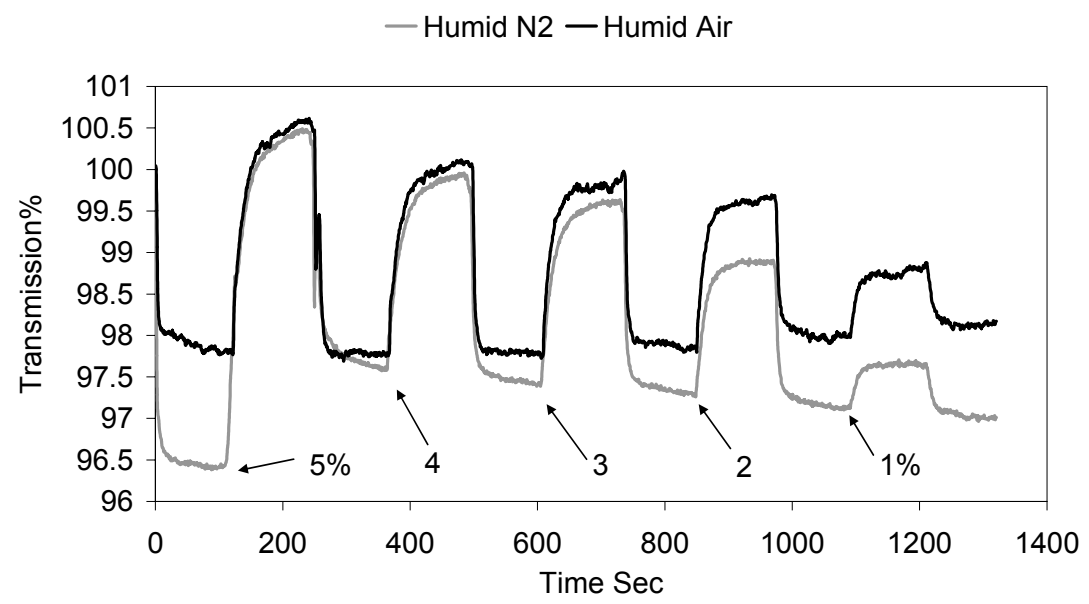

Figure 14 The sensor response to cyclic exposures of $1-5 \% \mathrm{H}_{2}$. 
By launching light perpendicular to the film, as shown in Figure 15(a), we demonstrated the film's sensitivity to hydrogen in transmission mode. Our idea is to miniaturize the sensor platform using integrated optic technology, and at the same time, to enhance sensitivity by increasing the optical pathlength.

\section{Waveguide Sensor Approach}

One method of accomplishing this might be to make the sensor film thicker, or even to cast the chemically-sensitive polymer into a "block" of material. However, in addition to causing problems in processing, this also would cause a marked increase in response time: diffusing hydrogen molecules into thick polymers takes much longer than the diffusion into thin films. The solution to this problem is to launch light into the sensor film "edge-on," as shown in Figure 15(b). In this configuration, light is confined to the plane of the film, and so travels through the width, rather than the thickness, of the film. Thus, the optical pathlength is increased without increasing the diffusion pathlength. Preliminary confirmation that we can fabricate such a waveguide from our sensor films is shown in Figure 16. A final refinement, which will increase sensitivity even more, is to further contain the light by limiting the lateral dimension of the optical waveguide, forming an integrated optic "channel," as shown in Figure 15(c). 


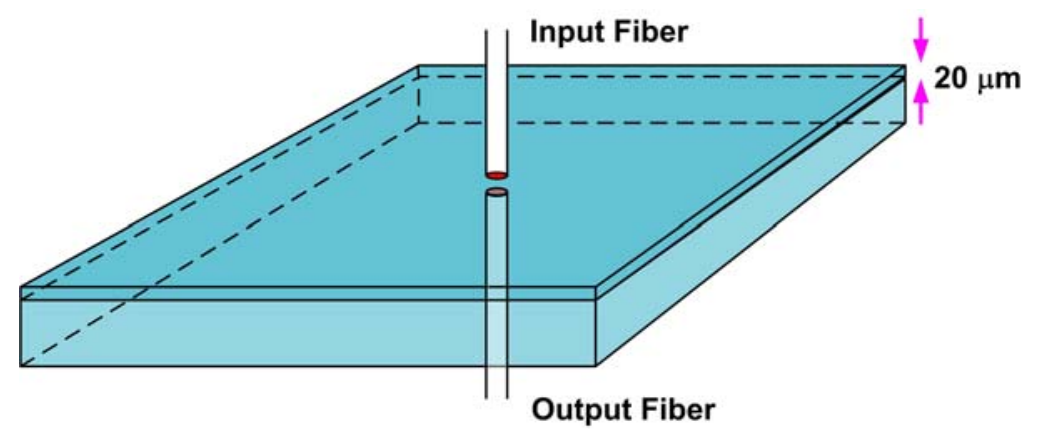

(a)

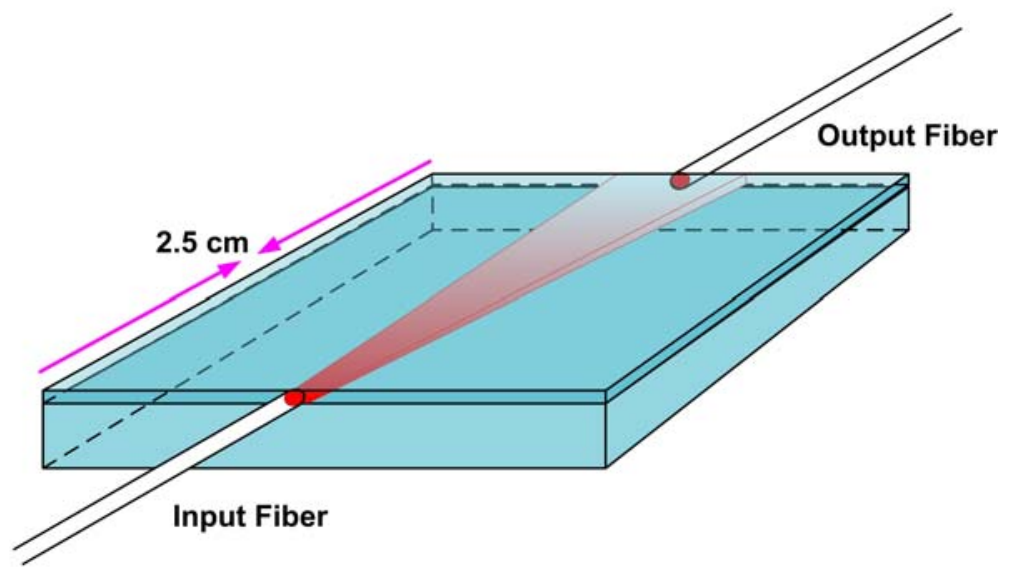

(b)

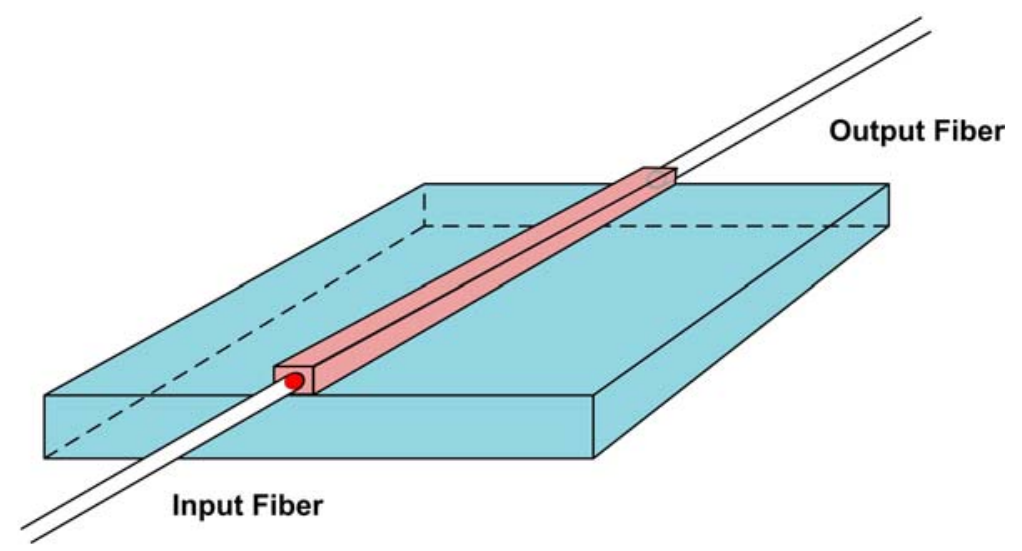

(c)

Figure 15 Thin film sensor geometries: a) perpendicular light launch. Pathlength equal to film thickness; b) in-plane light launch. Pathlength equal to film width; and c) "channel" waveguide. All of the input light reaches the output fiber. 


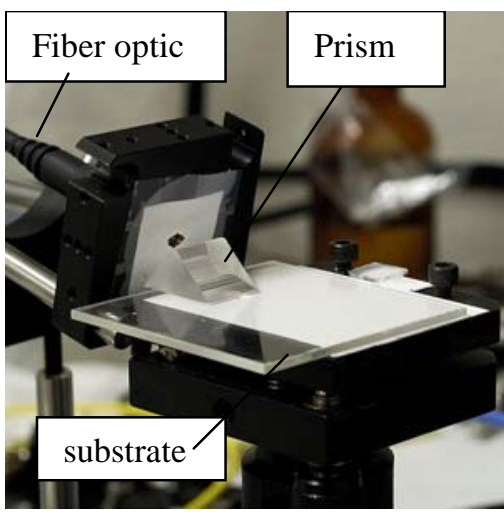

(a)

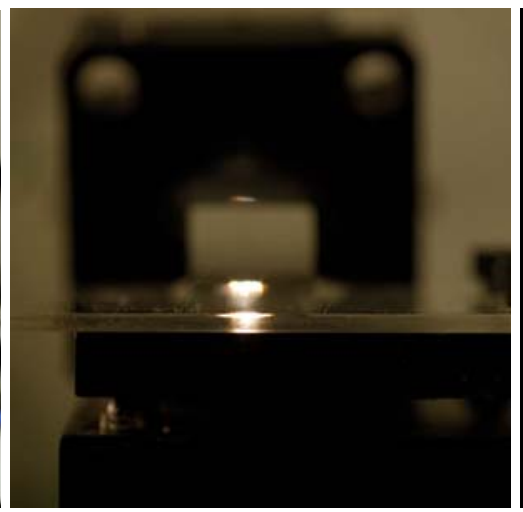

(b)

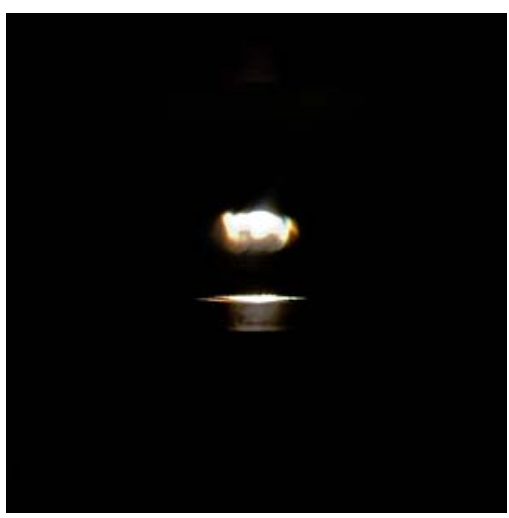

(c)

Figure 16 Edge-launched light guided by sensor film: (a) photograph of the experimental setup. The film is coated on a low index fused silica substrate. The index of the film is higher than silica hence the film acts as a waveguide. Light is directed into the "slab waveguide" (film) using an optical fiber and a prism. The optical fiber carries the light from the source, and the prism couples the light from the fiber into the film; (b) the near field view of the endface, opposite the prism. With the room light dim, it is easy to see the light coupling into and out of the film; (c) with the room light off, it is obvious that the film guides the light in a forward manner with beam expansion only within the film.

\subsubsection{Advanced Sensor Film Formulations}

An important finding is that the optical absorption of a sensor material at the operating wavelength has to be low, prior to chemical exposure, so that the material can be used as a waveguide. Immobilizing our current solution-based chemistry (D) yields a rather dark colored sensor that is difficult to utilize as a visible light waveguide longer than $10 \mathrm{~mm}$. However, we have developed two approaches to overcome this anomaly so that we can readily employ longer length waveguides. The first approach is to use the dark colored films in the near infrared, where the native absorption is low and the activity for hydrogen sensing is high. The second innovation is to replace the currently used metallic precursor in chemistry "D" with catalytic nanoparticles. The sensor films developed using this methodology are clear, with no initial color, but when exposed to hydrogen, they turn blue. Having no initial color makes the materials promising waveguide candidates. Hydrogen response results for a "perpendicular launch" (Figure 15(a)) into this film are shown in Figure 17. This material can be used in a waveguide format, which will increase the signal by an order of magnitude or more. 


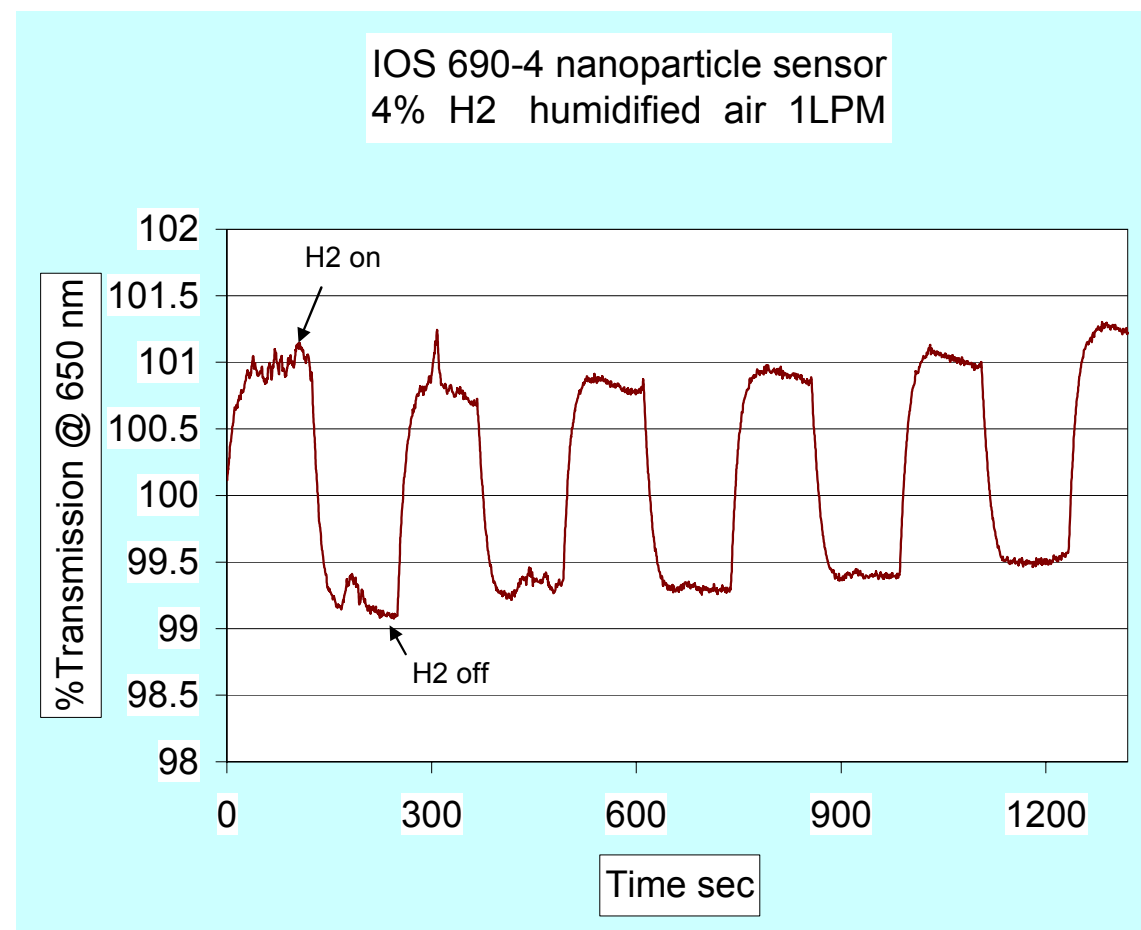

Figure 17 Polymer sensor material (utilizing nanoparticles) exposed to $4 \% \mathrm{H}_{2}$ cycling in $50 \%$ $\mathrm{RH}$ environment. Thickness of material is 20 microns.

The nanoparticles offer four distinct advantages: 1) they have a large surface area-to-volume ratio; 2) the metal nanoparticles are synthesized in the zero oxidation state and do not have to be reduced to that state at optical polymer-degrading high temperatures $\left(\sim 200^{\circ} \mathrm{C}\right)$, as do metal salts; 3) the nanoparticles, when formulated correctly, do not aggregate to yield the black color associated with highly concentrated metal; and 4) the nanoparticle diameter can be made small enough to allow us to incorporate them into the porous glass substrates for preliminary testing.

We have continued to develop solution-based indicator chemistries and incorporate these chemistries into polymeric substrates. We have demonstrated the feasibility of this approach by fabricating thin film polymeric sensors. Our goal is to have the option to use a polymer substrate rather than a porous glass substrate. We utilized the polymer substrate in a waveguide format in order to increase optical pathlength and enhance response. We have shown that we can fabricate polymer waveguides from our advanced sensor formulations; our results showed that light can be guided at the visible wavelength of 633nm (see Figure 18). We were also successful at guiding $810 \mathrm{~nm}$ light in these films. 

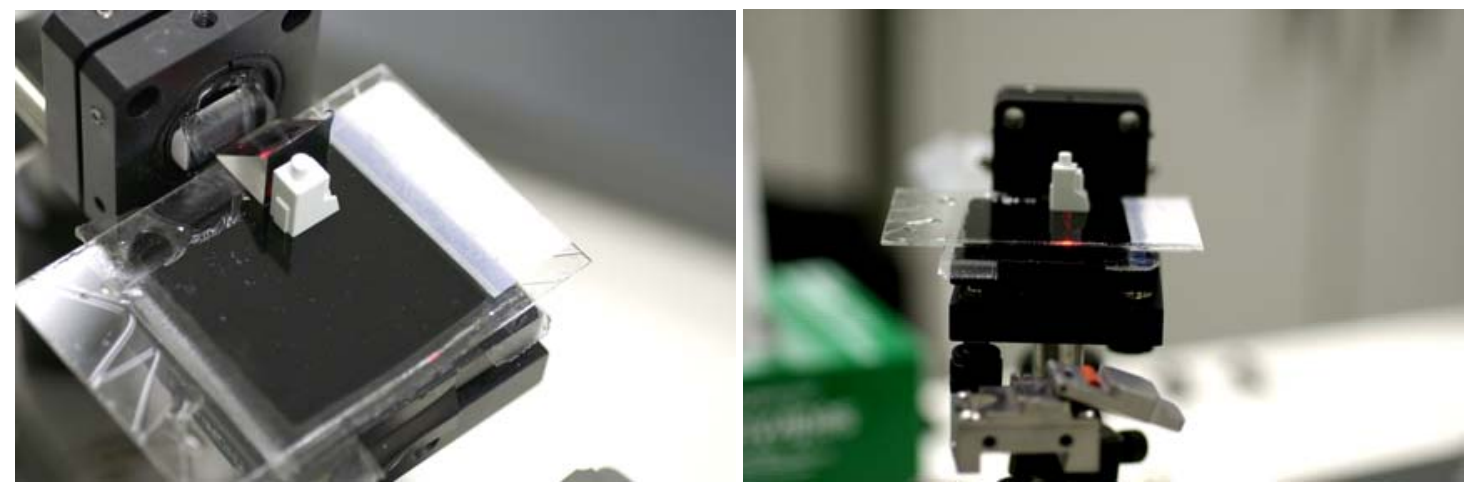

Figure 18 Experimental setup and example of light guided by sensor film: a 50 micron thick film is coated on a low index glass substrate. The index of the film is higher than the substrate, hence the film acts as a waveguide. Visible light $(633 \mathrm{~nm})$ is directed into the waveguide using an optical fiber and a prism. The optical fiber carries the light from the source, and the prism couples the light from the fiber into the film. A small non-transparent piece of plastic was placed in front of the prism to block scattered light so that a picture could be taken. The near field view of the endface, opposite the prism, shows the light coupling out of the film. The film guides the light in a forward manner, with beam expansion only within the film.

A potential difficulty in using catalytic nanoparticles is that their activity is short-lived. We found that sensor films made using nanoparticles last only 1-2 weeks. The dark colored sensor films, however, can last at least six months (see Figure 19). The short-lived activity of the nanoparticle-based sensor films is most likely due to the catalyst being poisoned by atmospheric pollutants, which is a known drawback. It is further known that thin film barrier coatings can minimize this effect.

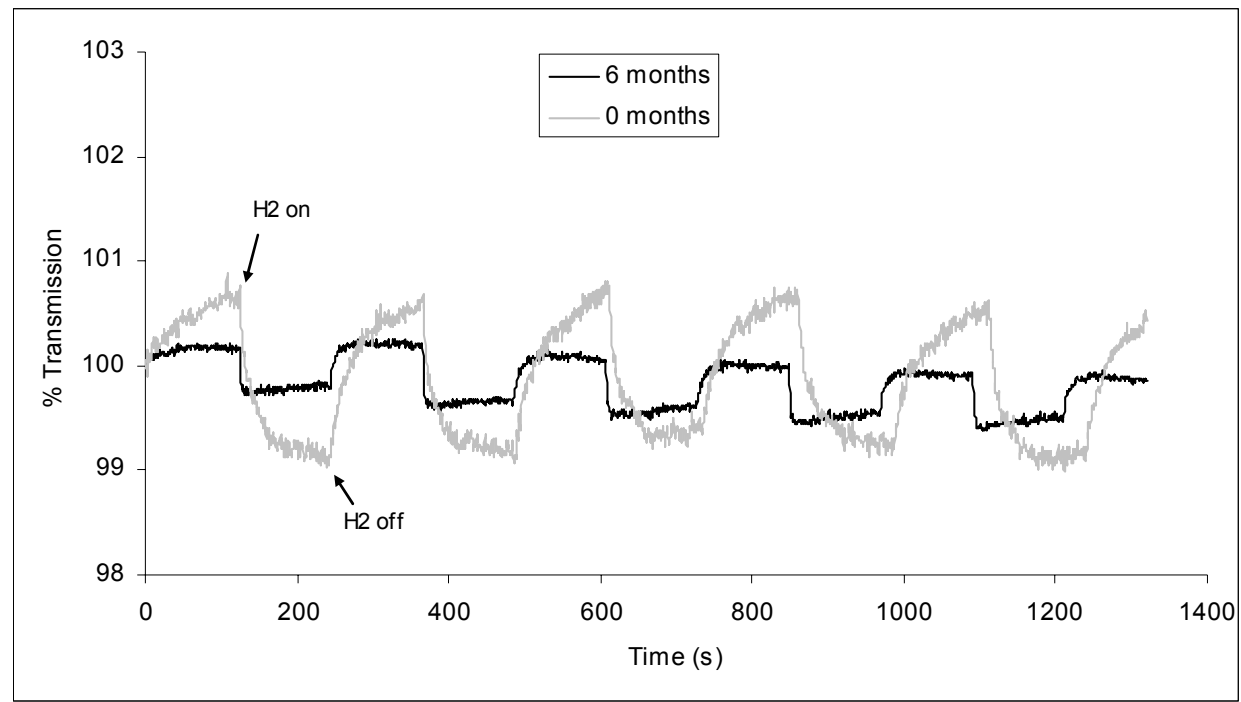

Figure 19 Transmission intensity (measured perpendicular to film) of $650 \mathrm{~nm}$ light taken from a polymer sensor when first made (gray) and after aged six months (black). The sensors were exposed to $4 \% \mathrm{H}_{2}$ cycling in $50 \% \mathrm{RH}$ environment. Thickness of the material is $\mathbf{2 0}$ microns.

We have demonstrated the sensitivity of our advanced polymer sensor materials below previously tested levels (i.e., 1\% hydrogen). A thin film of this material (ca. 40 microns thick) 
responds linearly between $4 \%$ and $0.5 \%$ hydrogen, as shown in Figure 20. The sensitivity of this material degrades slowly with time, but we anticipate a one year minimum lifetime. Our initial tests show that the material has a slight humidity dependent response to the hydrogen, and further work is needed to chemically modify the cross-linked polymer matrix to reduce or eliminate this interference. The polymer used in the sensor has excellent optical properties, and future work will be directed towards developing it into a fiber cladding or channel waveguide format, resulting in several orders of magnitude greater sensitivity. This enhanced sensitivity will be accompanied by a much longer lifetime, and reduced interference effects from oxygen or water vapor.

Polymer Film Sensor

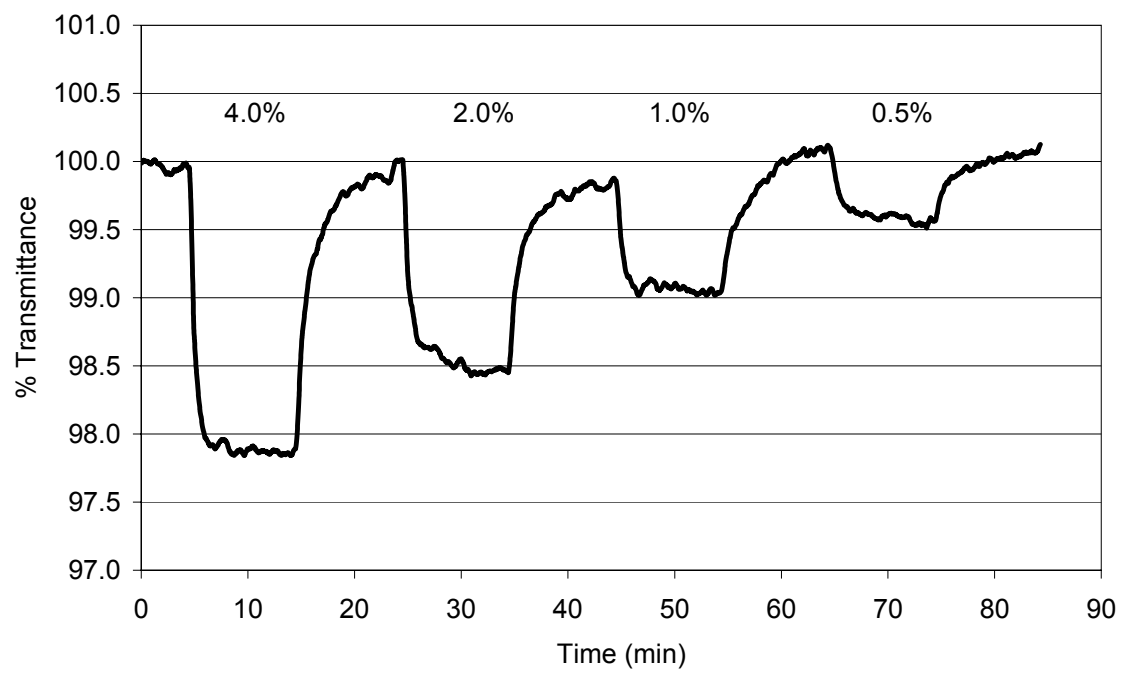

Figure 20 Polymer sensor response to hydrogen cycling of $4,2,1,0.5 \%$ (measured as sample transmittance of $650 \mathrm{~nm}$ light). Thickness of the material is 40 microns.

\subsection{Management and Marketing}

We established a consultant agreement with Mr. Jerald Cole, a well-known expert on hydrogen sensor technologies and the hydrogen economy. We also attended general meetings of the California Hydrogen Business Council (CHBC) at the South Coast Air Quality Management District (SCAQMD) headquarters. The CHBC is a vital link between hydrogen-technology developers, businesses, energy leaders, government, and infrastructure providers. We also met with Dr. Sigmund Gronich, which in turn led to a meeting with Dr. Patrick Davis to discuss our current results, and the prospect of future funding.

We attended the DOE sponsored hydrogen sensor workshop held in Washington DC. At this meeting, we had a scheduled project review meeting (with Carolyn Elam, Antonio Ruiz, and Patrick Davis) to discuss our current results, and the prospect of future funding. We also compiled the required presentation material and attended the Annual Merit Review Meeting in Arlington, VA. 


\subsection{SCHEDULE STATUS}

Project Management Plan

Tasks

1. Acquire reagents and substrate materials

2. Formulate sol-gel sensors from silicate and/or silicone reagents

3. Evaluate hydrogen diffusion in polymer materials

4. Evaluate various techniques to produce thin-film and/or slab sensors from advanced polymers and fabricate candidate sensors

5. Evaluate sensor resonse to hydrogen under inert conditions

6. Evaluate sensor performance and resisitance to moisture and oxygen

7. Incoporate new sensors in compact hydrogen detector and test system

8. Project management and reporting Milestones

1. The most promising candidate sensors will support homogenously distributed chemistry, while retaining optical transparency.

2. One or more candidate sensors will have good sensitivity, reponse time, and dynamic range to hydrogen gas at parts per million concentration in humid air with little or no dependence on humidity or oxgen concentration.

3. Optical hydrogen sensor with improved humidity and oxygen reistance Budget

$\%$ of total

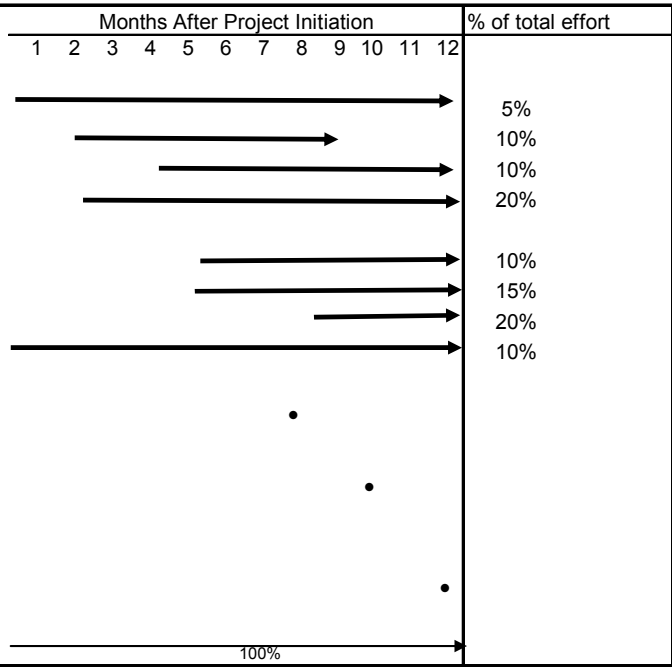

Completed- Solid black arrow

In Progress- dashed arrow

Pending- gray arrow

- Estimated completion date

- Actual completion date

\subsection{CONCLUSIONS}

This project yielded good progress in synthesizing two types of sensor materials. Novel sol-gel sensor materials that are responsive to hydrogen have been fabricated. Importantly, we have been successful at immobilizing our solution-based chemical indicators in advanced polymeric materials. Thin films were fabricated of the polymer materials, and our tests showed that they easily detect $1 \%$ hydrogen. It was proven that these novel polymeric materials are applicable in a thin film waveguide sensor format. The polymeric waveguide sensor approach is highly synergistic with current IOS projects, which will facilitate the accelerated commercialization of these sensors.

We made excellent progress in developing sensors that are less susceptible to humidity interference. We have arrived at three final sensor types that support homogenously distributed chemistry, and that have optical transparency. By achieving this goal, we successfully met Milestone 1. The selected formulations are as follows:

- Porous Glass Substrate

1. IOS original sensor format with moisture barrier layer;

2. IOS sensor formatted using nanoparticles and moisture barrier layer; and - Polymeric Substrate

3. IOS novel polymeric sensor format utilizing nanoparticles.

We determined that the polymer-coated porous glass sensor responds to a sub-percentage concentration $(0.5 \%)$ of hydrogen in a 95\% humid environment. With this prototype sensor substrate, we satisfied the criteria of Milestone 2. We successfully fabricated a waveguide using our polymeric sensor substrate, and used it to carry both visible and near infrared light. The polymer thin film sensor also responds to $0.5 \%$ hydrogen. Further tests and optimizations are 
needed to reduce the interference caused by humidity. A small, lightweight eight-channel sensor unit, which provides a multiplexed capability, was manufactured, satisfying Milestone 3. This unit can be readily altered into a more sophisticated lock-in detection unit. During the course of this project, we have successfully met all project milestones. 